\title{
Automatic generation of objective footprint outlines
}

\author{
Jens N Lallensack ${ }^{\text {Corresp. } 1}$ \\ ${ }^{1}$ Division of Paleontology, Institute of Geosciences, University of Bonn, Bonn, Germany \\ Corresponding Author: Jens N Lallensack \\ Email address: jens.lallensack@uni-bonn.de
}

The objective definition of footprint margins poses a central problem in ichnology. The transition from the footprint to the surrounding sediment is often continuous, and the footprint wall complex, requiring interpolation, approximation, and a priori assumptions about trackmaker anatomy to arrive at feasible interpretations of footprint shapes. The degree of subjectivity of such interpretations is substantial, and outlines produced by separate researchers can differ greatly. As a consequence, statistical shape analysis, regardless if based on linear and angular measurements or on the shape as a whole, are neither fully repeatable nor objective.

Here I present an algorithm implemented in the programming environment $\mathrm{R}$ that is able to generate continuous footprint outlines based on three-dimensional models-fully automatically, objectively, and repeatable. The approach, which is based on contour lines extracted from the model, traces the outline at the point where the slope of the track wall is steepest. An option for automatic landmark placement is implemented for tridactyl footprints. A case study was carried out on 13 footprints of a single trackway of a theropod trackmaker from the Lower Cretaceous of Münchehagen, Germany. Analysis of the landmark coordinates returned by the script did reproduce statistical results published in an earlier study that was based on human-made interpretative drawings, demonstrating the applicability of the present method for the objective and quantitative shape analysis of tracks. Although faint anatomical details are not always recorded and features not related to the foot anatomy may be included, the generated outlines tend to correspond with human-made interpretative drawings regarding the overall shape. While not suited as a full replacement of interpretative drawings, these generated outlines may be used as an objective basis for such interpretations. 


\section{Automatic generation of objective footprint outlines}

2 Jens N. Lallensack ${ }^{1}$

$3{ }^{1}$ Division of Paleontology, Institute of Geosciences, University of Bonn, Bonn, Germany.

4

5 Corresponding Author:

6 Jens N. Lallensack ${ }^{1}$

7 Nussallee 8, 53115 Bonn

8 Email address: jens.lallensack@gmail.com

\section{Abstract}

11 The objective definition of footprint margins poses a central problem in ichnology. The transition from the footprint to the surrounding sediment is often continuous, and the footprint wall complex, requiring interpolation, approximation, and a priori assumptions about trackmaker anatomy to arrive at feasible interpretations of footprint shapes. The degree of subjectivity of such interpretations is substantial, and outlines produced by separate researchers can differ greatly. As a consequence, statistical shape analysis, regardless if based on linear and angular measurements or on the shape as a whole, are neither fully repeatable nor objective.

Here I present an algorithm implemented in the programming environment $\mathrm{R}$ that is able to generate continuous footprint outlines based on three-dimensional models-fully automatically, objectively, and repeatable. The approach, which is based on contour lines extracted from the model, traces the outline at the point where the slope of the track wall is steepest. An option for automatic landmark placement is implemented for tridactyl footprints. A case study was carried out on 13 footprints of a single trackway of a theropod trackmaker from the Lower Cretaceous of Münchehagen, Germany. Analysis of the landmark coordinates returned by the script did reproduce statistical results published in an earlier study that was based on human-made interpretative drawings, demonstrating the applicability of the present method for the objective and quantitative shape analysis of tracks. Although faint anatomical details are not always recorded and features not related to the foot anatomy may be included, the generated outlines tend to correspond with human-made interpretative drawings regarding the overall shape. While not suited as a full replacement of interpretative drawings, these generated outlines may be used as an objective basis for such interpretations. 


\section{Introduction}

34 Fossil footprints are an important supplement to the body fossil record, given their abundance and nature as life traces that directly record behavior and locomotion. Yet, the potential of analyses combining footprint and body fossil data is not yet exhausted, partly due to the slow advancement of objective and quantitative methodology in ichnology. A central problem in applying such methods to footprint data is the inability to objectively define the margins of a footprint, especially when the footprint indistinctly grades into the surrounding sediment. Falkingham (2016) demonstrated that the length of a footprint can vary as much as $27 \%$ depending on the height level chosen for measurement. Various criteria for the identification of the footprint margin have been proposed, including the point of inflexion of the footprint wall, the minimum outline, and the selection of a single contour line, amongst others (Falkingham, 2016). However, none of these criteria is unambiguously applicable to a wider range of different footprints, which typically show multiple inflexion points and often do not show distinct minimum outlines (Falkingham, 2016; Lallensack, van Heteren \& Wings, 2016). Adding to the problem, the vast majority of ichnological publications does not specify the criteria used for defining the footprint margins. The inability to objectively define footprint margins is highly problematic especially when quantitative methods are to be applied to analyze footprint shape, since such analyses can only generate fully objective results when based on objective input data (Falkingham, 2016).

The problem persists when not only linear and angular measurements but a single, twodimensional outline abstracting the whole shape of the footprint is to be extracted. The outlines of one and the same footprint, when drawn by separate researchers, can differ considerably from each other (Thulborn, 1990), which repeatedly led to calls for caution in interpreting such data (e.g., Sarjeant, 1975; Thulborn, 1990; Falkingham, 2010, 2016). Furthermore, the high degree of simplification of two-dimensional outlines has been criticized, proposing that the full threedimensional profile should be retained instead (e.g., Ishigaki \& Fujisaki, 1989; Belvedere et al., 2018). Nevertheless, outline drawings remain the most widely used means for distributing footprint shape data, also because most anatomical information of the footprint is contained in its outline. Problematically, it is often not possible or desirable to excavate and archive footprints in museum collections, which is why material is often difficult to access or get degraded by weathering (Bennett et al., 2013). Ichnologists, therefore, are in many cases forced to rely on subjective outline drawings presented in the literature for ichnotaxonomic attributions and comparisons with relevant material.

Recent efforts to increase objectivity in footprint research rely on 3D-digitization techniques, most importantly photogrammetry, which allows for the fast and cost-effective capturing of footprint morphologies in high resolution (e.g., Falkingham, 2012; Mallison \& Wings, 2014; Matthews, Noble \& Breithaupt, 2016). A relatively new set of methods in the field, these techniques promise to solve critical problems of collection and dissemination of footprint data, and have been recently accepted as best practice in the documentation of fossil footprints 
71 (Falkingham et al., 2018). Thus, the availability of such models can be expected to further 72 increase in the future.

73 Although a number of methods for the analysis of footprint shapes exist, none can effectively

74 solve or circumvent the problem of the definition of footprint margins. Comparative approaches

75 using 3D geometric morphometrics (Bennett et al., 2016; e.g., Belvedere et al., 2018) will include

76 both the footprint and the surrounding sediment unless the footprint margin has been defined a

77 priori. Therefore, such methods are feasible only when foot posture, most importantly the

78 interdigital angles, is constant, as otherwise regions of the footprint may get averaged with

79 surrounding sediment. Furthermore, the registration of the separate footprints still requires user-

80 defined landmarks, which often cannot be placed unequivocally in the absence of an objective

81 definition of the footprint margin.

82 The algorithm presented herein allows for the fully objective and automatic generation of

83 continuous outlines based on 3D surface models of footprints. The method relies on the steepness

84 of the footprint slope (i.e., the inflexion point of the footprint wall), the probably most commonly

85 used criterium for the definition of footprint margins (Ishigaki \& Fujisaki, 1989).

86

87

88

89

90

91

92

93

94

95

96

97

98

99

100

101

102

103

104

105

106

107

\section{Methods}

The algorithm presented herein (source code provided as data S1), implemented in the programming environment $\mathrm{R}$, allows for the fully objective and automatic generation of continuous outlines based on 3D surface models of footprints. Many required functions have been already implemented in the wealth of packages available for $\mathrm{R}$; these were used whenever possible, reducing the script to approximately 650 lines of code. The implemented $\mathrm{R}$ function, named "oboutline", will perform the import of the 3D model and the calculation of the outline automatically in a single step, without requiring additional human input. The output of the script consists of vector plots (.svg) including the objective outline, the coordinates of the objective outline (.csv), and a .ply file of the input model fitted to the horizontal plane. When running with support for tridactyl footprints enabled, the script will additionally return landmark coordinates, a resampled version of the objective outline with homologous points, and a vector plot including the landmarks.

\section{Model import, orientation, and contour line generation}

Import of 3D-models is achieved using the vcgImport function of the Rvcg package (Schlager, 2017), which supports commonly used formats including the widely used PLY. The supplied 3Dmodel should contain only a single complete footprint as well as a margin of surrounding surface. The exact extent of the surrounding margin will not alter results except when an increased margin size includes additional large-scale continuous contours that can be mistakenly recognized as forming part of the footprint margin. After import, the script rotates the point cloud of the extracted vertex point xyz-coordinates to fit the horizontal plane (i.e., the tracking surface on which the animal walked) by employing principal component analysis (PCA) on the three 
108 variables $(\mathrm{x}, \mathrm{y}, \mathrm{z})$. The PCA fits three orthogonal axes (PC1-3) to the point cloud. PC1 is defined 109 as the axis of greatest variation, with PC2 and PC3 capturing successively less variation. In most 110 situations, PC1 and PC2 will represent the horizontal plane (the plane of greatest variation), and 111 PC3 the relief (i.e., the deviation from the horizontal plane). Problematically, the point cloud can 112 get mirror-inverted during PCA fitting as the signs of the columns of the rotation matrix are 113 arbitrary, a problem also occurring in respective implementations in 3D-mesh software like 114 Meshlab (tested with version v2016.12) or CloudCompare (tested with version 2.9). The present 115 script calculates the Procrustes distance (a measure of shape difference) of a subset of points of 116 the model before and after the PCA fit, and will mirror back when detecting a significant 117 difference. If the footprint is a cast (convex hyporelief) rather than a mold (concave epirelief), it 118 will be automatically mirrored into a mold. The scale of the input 3D model is preserved 119 throughout the process; measurements of the generated outline can be taken from both the plots 120 and coordinates returned by the script.

121 All subsequent computations are based on contour lines of 30 equally spaced height levels 122 extracted from the point cloud using the "getContourLines" function of the contoureR R package 123 (Hamilton, 2015, Fig. 1B). Contour lines reduce the complex three-dimensional problem to a 124 simpler and easier-to-handle two-dimensional one, and form the natural basis for 2D footprint 125 outlines. Before the objective outline can be extracted, a number of additional steps are required, 126 including 1) the removal of contours not representative for the footprint wall and 2) the 127 establishment of homology between the points of separate contours.

128 Contours not representative for the footprint wall are excluded based on simple criteria. First, all 129 open contours are removed, eliminating structures that continue beyond the margins of the model. 130 Second, only the longest contour of each height level is selected and kept, respectively, with all 131 others removed. This results in a stack of continuous contours, with one contour per height level.

132 Third, all contours less than 50\% of the length of the longest contour are removed, while assuring 133 that no gaps within the stack are being created. This approach eliminates smaller structures within 134 the footprint that are unlikely to contain relevant information on the footprint wall (Fig. 1C). An 135 option is implemented that allows for processing multiple impression per model, which is useful 136 in cases where the footprint is not defined by a single outline. For each additional impression, the 137 contour selecting procedure is repeated with the contours selected for the previous stacks 138 excluded.

139 The resulting stack of contours may still include a number of contours that convey little or no 140 information on the footprint wall, including roundish contours around the actual footprint. To 141 eliminate these contours as well, and to limit the height range under consideration, areas of all 142 contours are computed as a measure of form difference. Starting from the middle contour of the 143 stack, the differences in area of each contour with its next lowest (or highest) neighbor are 144 compared; if the difference in area between two contours exceeds a pre-defined threshold-value, 145 the upper (or lower) of this contour and all following contours are removed (Fig. 1D). Separate 146 threshold-values are defined for the lower and the upper half of the stack. An option for adjusting 
147 these values is available, allowing to influence how many lower or upper contours are to be

148 included, possibly changing the height level of the resulting calculated outline. All footprints

149 presented in this work were calculated using the default parameters.

\section{Homologization of contours}

151 Even if the starting point would correspond between all contours and if each contour would 152 contain an equal number of equidistant points (requirements not fulfilled a priori), the individual 153 points of the separate contours would tend to deviate from each other when far from the starting 154 point, as the shapes of the contours are not identical. For this reason, when producing a simple 155 mean shape, points would be averaged obliquely rather than perpendicularly to the footprint wall, 156 leading to erroneous results. The implemented solution detects a number of "bottlenecks"-pairs 157 of points with minimum distance between the innermost and outermost contour. Points forming 158 the bottleneck will be considered homologous (define a line that is assumed to be perpendicular 159 to the footprint wall), and the points in-between the bottlenecks will be interpolated by 160 resampling.

161 First, all contours are resampled to the same number of equidistant points, using 500 points per

162 default. The resulting contours can be variously oriented clockwise or counter-clockwise; 163 contours are reversed accordingly to achieve uniform orientations. Second, Euclidean distances 164 between all possible pairs of the inner and outer contour of the stack are calculated and stored in 165 a matrix with the dimensions $\mathrm{n} \times \mathrm{n}$. The pair with the minimum distance, the first bottleneck, is 166 then extracted, and those points of the intermediate contours are detected that are closest to a line 167 defined by the bottleneck points. The resulting set of homologous points is then defined as the 168 starting point of the contours. Third, additional bottlenecks are detected to establish homology. 169 The implemented algorithm first detects a second bottleneck on the side of the footprint opposite 170 to the first bottleneck; two additional bottlenecks are then found on each side between the first 171 and second bottleneck. More bottlenecks are detected within the intermediate sections if the latter 172 are long enough. In all cases, bottlenecks in proximity to existing bottlenecks are prevented, 173 assuring a roughly equal distribution of bottlenecks along the outline (Fig. 1D). Finally, the 174 individual sections between the bottlenecks are resampled to equal numbers of equidistant points, 175 which can now be considered homologous.

\section{Tracing of the steepest slope}

177 The objective outline will be traced along the steepest slope of the track wall. For each point 178 within each set of homologous points, the minimum distance between the neighboring contours is 179 measured. A set of homologous points is not always fully perpendicular to the footprint wall, 180 especially when the section between the bottleneck points is long and contours differ much in 181 orientation. For this reason, the algorithm does not simply compute the distances within the set of 182 homologous points, but the distances between each of the homologous points and all nearby 183 points within and outside of the set. The steepest slope computed this way (Fig. 1E) is seldom 
184 continuous along the whole outline, but rather tends to fade out and continue on a different height

185 level, frequently leading to abrupt steps in the outline that are obviously incompatible with

186 human interpretations. For this reason, the algorithm does not only detect the steepest point, but

187 takes into account the steepness at all other points. Then, the final coordinate of the steepest slope

188 is computed as the weighted arithmetic mean of all these points. Points will receive equal weight

189 only when the steepness is equal; the lower the steepness compared to the steepest slope, the less

190 weight is given.

191 The continuous and smooth final outline is produced by applying an approximating

192 function to the resulting set of points. Of various tested options, including B-splines and

193 Bézier curves, elliptic Fourier transforms were found to produce results most similar to

194 those expected from a human interpreter (Fig. 1F, 2). Elliptic Fourier transforms are

195 performed using the "efourier” function of the R package Momocs (Bonhomme et al.,

196 2014) using 25 harmonics and 10 smoothing iterations.

197 As the resulting objective outline and contour stack will be rotated arbitrarily, an algorithm

198 attempts to rotate both outline and stack into an upright orientation. This algorithm is based on

199 the assumption that digit impressions are facing upwards and require a longer contour segment

200 (i.e., more equidistant points) to be described. In a first step, the centroid, or center of mass, of

201 the outline is computed. Then, the length of the part of the outline above the centroid is calculated

202 for all rotation angles $\left(1-360^{\circ}\right)$, and the outline rotated according to the angle that maximizes this

203 length.

\section{Automatic landmark placement}

205 The script, as described above, can be applied to any kind of depression, as no a priori

206 assumptions on trackmaker anatomy are introduced. Such assumptions are required when linear

207 and angular measurements are to be extracted or when different outlines are to be aligned for

208 quantitative shape analysis. Therefore, a function is included that returns landmark coordinates

209 for tridactyl footprints, which are the most common dinosaur footprints and one of the most

210 common footprint types in general. The algorithm first detects and separates the three digit

211 impressions, assuming that the outline was correctly rotated in a more-or-less upright orientation

212 in the previous step. Then, the rotation angle of the outline is further refined by considering the

213 central digit impression only, which is commonly considered to approximate the mid-axis of the

214 footprint (Leonardi et al., 1987). A total of six landmarks are defined as in (Lallensack, van

215 Heteren \& Wings, 2016), on the tips of the three digit impressions, on the two hypex points, and

216 on the heel. The landmarks on the tips of the digit impressions are defined as the distal ends of

217 the digital axes; this definition reduces the influence of claw impressions, which may vary

218 strongly depending on preservation and behavior (Lallensack, van Heteren \& Wings, 2016). The

219 digital axes were computed by rotating the respective digit into the upright position and taking

220 the mean of the $\mathrm{x}$-coordinates; the intersection with the outline was found using the rgeos $\mathrm{R}$

221 package (Bivand \& Rundel, 2018). The two hypex points were computed by finding the lowest 
222 point between the enclosing digit impressions relative to a line connecting the landmarks at the

223 tips of these digit impressions. The landmark on the heel region is the intersection of the axis of

224 the central digit impression and the proximal margin of the outline.

\section{Case study and sensitivity analysis}

226 The applicability of the presented approach for the quantitative analysis of footprints was tested

227

228

229

230

231

232

233

234

235

236

237

238

239

240

241

242

243

244

245

246

247

248

249

250

251

252

253

254

255

256

257 on 13 footprints pertaining of a single theropod trackway (T3) from the Lower Cretaceous of Münchehagen, Germany (3D-models are provided as data S2). Geometric morphometric analysis of the same footprints was conducted in earlier studies based on interpretive drawings (Lallensack, van Heteren \& Wings, 2016; Wings, Lallensack \& Mallison, 2016), suggesting that 1) the landmarks on the hypex positions and on the heel are more variable than the landmarks on the digital tips, and that 2) the lateral hypex is more variable than the medial hypex. In the present case study, the calculated objective outlines were aligned using Generalized Procrustes Analysis (GPA) with the geomorph R package (Adams, Collyer \& Kaliontzopoulou, 2018). The mean shape of the aligned shapes was then compared with the meanshape published by (Lallensack, van Heteren \& Wings, 2016). This is expected to reveal potential systematic differences between the traditional interpretive approach and the automated approach presented herein. Furthermore, landmarks are automatically extracted from all 13 generated outlines as described above, and their variability in y-direction (parallel to the footprint mid-axis) computed, in the same way as has been done in (Lallensack, van Heteren \& Wings, 2016) based on interpretive outlines.

\section{Results and discussion}

\section{Limitations}

Human-made interpretational footprint drawings aim to capture as much information about the trackmaker anatomy as preserved. Although slope steepness is the most important criterion, the steepest slope will frequently fade out along the footprint wall to continue at a different height level, making interpolation unavoidable. Furthermore, humans tend to make a priori assumptions about trackmaker anatomy when producing the outlines, which allows them to take into account extramorphological (unrelated to the foot anatomy) features and include anatomical features of interest such as digital pad impressions and claw marks.

The present algorithm is successful in detecting and interpolating outlines even when the steepest parts of the slope are indistinct (Fig. 2). It does currently not include a priori assumptions that would emphasize anatomically important details and account for extramorphological features, which keeps the algorithm simple and predictable, and applicable to a wide range of footprint types. The broad applicability is demonstrated in Fig. 2C with a hominin footprint from the famous Laetoli tracksite of Tanzania (Leakey \& Hay, 1979). In the absence of a priori assumptions, however, the outlines expectedly tend to provide less information on the presumed foot anatomy than interpretative drawings, and may be unusable in cases where track morphology 
258 is obscured by extramorphological features. Artifacts caused by a crack running transversally

259 through the central digit impression are shown in Fig. 2A for a medium-sized theropod footprint

260 from the Late Jurassic Courtedoux-Bois de Sylleux tracksite of Switzerland (Castanera et al., 261 2018).

262 Additional limitations currently arise from the necessity to limit the vertical extent of the contour

263 stack, which may exclude relevant anatomical features not captured by the selected contours.

264 Furthermore, the described approach requires that impressions can be described by single

265 contours, which is not the case in all cases, especially when the footprint is very shallow and

266 indistinct. However, a preliminary option is implemented to process footprints that are comprised

267 of more than one impression, as demonstrated with an ornithopod footprint attributed to

268 Caririchnium kyoungsookimi from the Jindong Formation in Goseong County, South Korea (Fig.

269 2B, 3D data provided by Anthony Romilio). This footprint is relatively shallow (maximum depth

270 is $3.8 \%$ of maximum length of model) and comprises three separate impressions.

\section{Qualitative comparisons with human-made outlines}

273 The similarity of the generated outlines with human-made interpretations is demonstrated on five

274 fossil footprints that have been previously published in the literature (Fig. 2D-I). All five

275 examples are compared with published interpretative drawings that had been produced using the

276 same 3D-models; all examples, as is the case for examples used elsewhere in this work, were

277 processed using the same script version and parameters. Footprints T3/47, T3/37, and I1-35 (Fig.

278 2D-F, respectively) come from the Lower Cretaceous of Münchehagen, Germany (Lallensack,

279 van Heteren \& Wings, 2016; Wings, Lallensack \& Mallison, 2016). All three footprints were left

280 by the right foot. T3/47 and T3/37 were part of a larger theropod and I1/35 (Fig. 2F) of an

281 ornithopod trackway. The objective outlines (red continuous lines) are generally in accordance

282 with the interpretational drawings published in (Lallensack, van Heteren \& Wings, 2016) (dotted

283 blue lines). However, the sediment bars between the digital impressions tend to be less extensive

284 (e.g., Fig2E-F), and digital impression IV in T3/47 is somewhat abbreviated in the objective

285 outline due to sediment infilling in the distal tip of the impression. The generally good match

286 between the generated outlines presented herein and the interpretive ones of (Lallensack, van

287 Heteren \& Wings, 2016) may be partly due to the use of similar criteria for the definition of the

288 track margin, which are discussed in detail in the latter study, most importantly the criterion of

289 the steepest slope.

290 Tracks 5 and 6 of QM F10322 (Fig. 2G-H) are the left and right pedal impressions of a large

291 tridactyl trackway from the Upper Cretaceous of Lark Quarry in Queensland, Australia (Thulborn

292 \& Wade, 1984; Romilio \& Salisbury, 2011, 2014). The 3D-models, based on photographs of the

293 in situ specimen taken in 2013, were provided by Anthony Romilio. The trackway became

294 famous after it was suggested to have been left by a large theropod causing a dinosaur stampede

295 (Thulborn \& Wade, 1984), a hypothesis that has been discussed controversially in recent years 
296 (Romilio \& Salisbury, 2011, 2014; Thulborn, 2013, 2017; Romilio, Tucker \& Salisbury, 2013),

297 with the identification of the large tridactyl trackmaker as either a theropod or an ornithopod

298 constituting a major point of disagreement. This discussion is instructive in showing how much

299 interpretative outlines can differ when produced by separate researchers with different

300 preconceptions about the responsible trackmaker taxon. Well aware of the subjectivity problem,

301 Romilio \& Salisbury (2014, fig. 7F, 8F) did not produce traditional outline drawings but selected

302 a single contour line they considered representative. Still, this approach is not completely

303 objective, as separate contours can differ greatly in shape and dimensions (Falkingham, 2016).

304 The calculated outlines presented herein correspond well with those of Romilio \& Salisbury

305 (2014) (Fig. 2G-H). However, they differ considerably from outlines of the same imprints

306 presented by Thulborn \& Wade (1984, plate 17), and especially from a more recent interpretation

307 of track 5 by Thulborn (2017, fig. 5 (3)), which is redrawn here for comparison (Fig. 2I). Track 5

308 (Fig. 2H-I) is a prime example of how different interpretational drawings can be when based on

309 fundamentally different assumptions. Given their interpretational nature, it is, on principle, not

310 possible to discard one of these disparate interpretations as incorrect (Falkingham, 2016).

311 Furthermore, the good correspondence of the generated outline with the outline presented by

312 (Romilio \& Salisbury, 2014) could be partly due to the fact that both outlines were produced

313 based on contour lines, while the differing outline of (Thulborn, 2017) was traced on a

314 photograph. Nevertheless, the presented approach may offer a standard to which interpretive

315 outlines can be compared. It furthermore allows for an objective qualitative comparison of

316 different footprints, as generated outlines would be free of preconceived assumptions on the

317 trackmaker taxon and computed using the same parameters. Although not a replacement for

318 interpretive outlines given the limitations outlined above, generated outlines may be used in

319 combination with interpretive ones, and may form the objective basis for the production of the

320 latter.

\section{Uses in quantitative shape analysis}

322 All 13 footprints of the T3 trackway included in the original geometric morphometric analysis of 323 (Lallensack, van Heteren \& Wings, 2016; Wings, Lallensack \& Mallison, 2016) were processed

324 using the same program version and parameters (Fig. S3), and analyzed following the protocol

325 provided by the mentioned studies. The mean shape derived from the Procrustes-aligned

326 generated outlines closely matches the previously published mean shape that was based on

327 interpretive outlines of the same tracks (Fig. 3C). This indicates that, at least in this case,

328 systematic differences in generated and interpretive outlines are minor. Analysis of the variability

329 of the six landmarks (as indicated in Fig. 3A), however, reveals more substantial differences (Fig.

330 3D). The objective approach confirms that the lateral and medial hypex (landmarks 2 and 4) are

331 more variable than the remaining landmarks. However, the previously published observation that

332 the heel is highly variable is not supported. Furthermore, the central digit (landmark 3) is

333 markedly more variable according to the objective approach. This is partly due to sediment

334 infilling of the distal end of this digit impression (an extramorphological feature) in one of the 
335 footprints, which is not taken into account by the objective approach (Fig. 3B). This case study

336 demonstrates that generated outlines can be used to objectively reproduce results derived from

337 interpretive outlines, although the possible influence of extramorphological features and other

338 potential errors in the objective outlines need to be taken into account.

339 To test for repeatability of results generated by the present approach, one model (track 6 of QM

340 F10322) was saved at successively lower resolutions using MeshLab, and the resulting outlines

341 were compared (Fig. 3E-F). Observed differences resulting from the separate model resolutions

342 are found to be negligible, even when the highest resolution (Fig. 3E; 196,236 faces, 9.4 MiB) is

343 compared with the lowest tested resolution (Fig 3F; 5000 faces, $246.2 \mathrm{KiB}$ ).

\section{Conclusions}

345 The lack of widely applicable, objective means for defining the footprint margin is among the 346 most vexing problems in the research of fossil footprints. The present algorithm automatically

347 generates continuous objective footprint outlines by employing the criterium of the steepest

348 slope. In contrast to human-made interpretive outlines, these generated outlines allow for

349 analyses that are fully reproducible and free of interpretational bias, as complete samples can be

350 processed using the same mathematically defined criteria. Although the generated outlines tend to

351 correspond well with interpretive outlines, extramorphological features unrelated to the foot

352 anatomy may be incorporated, and anatomical detail not captured by the steepest slope may be

353 excluded. While not a fully appropriate replacement for human-made drawings in most cases,

354 computed outlines may be used as an objective basis for the production of interpretational

355 drawings, and allow for objective qualitative comparisons. Most importantly, the approach paves

356 the way for fully objective quantitative analyses of footprint shapes.

\section{Acknowledgments}

358 I like to thank Michael Buchwitz, Karl Bernhardi and Vincent Bonhomme for discussions, ideas,

359 and help with writing the presented software. Anthony Romilio and Matthew Bennett provided

360 additional 3D models that were important in testing this software.

\section{References}

Adams DC, Collyer ML, Kaliontzopoulou A. 2018. Geomorph: Software for geometric morphometric analyses. $R$ package version 3.0.6. https://cran.r-project.org/package=geomorph.

Belvedere M, Bennett MR, Marty D, Budka M, Reynolds SC, Bakirov R. 2018. Stat-tracks and mediotypes: powerful tools for modern ichnology based on 3D models. PeerJ 6:e4247. 
Bennett MR, Falkingham P, Morse SA, Bates K, Crompton RH. 2013. Preserving the impossible: Conservation of soft-sediment hominin footprint sites and strategies for three-dimensional digital data capture. PLoS ONE 8:e60755. DOI: doi:10.1371/journal.pone.0060755.

Bennett MR, Reynolds SC, Morse SA, Budka M. 2016. Laetoli’s lost tracks: 3D generated mean shape and missing footprints. Scientific reports 6:1-8. DOI: 10.1038/srep21916.

Bivand R, Rundel C. 2018. rgeos: Interface to Geometry Engine - Open Source ('GEOS').

Bonhomme V, Picq S, Gaucherel C, Claude J. 2014. Momocs: Outline Analysis Using R. Journal of Statistical Software 56:1-24.

Castanera D, Belvedere M, Marty D, Paratte G, Lapaire-Cattin M, Lovis C, Meyer CA. 2018. A walk in the maze: variation in Late Jurassic tridactyl dinosaur tracks from the Swiss Jura Mountains (NW Switzerland). PeerJ 6:e4579; DOI 10.7717/peerj.4579

Falkingham PL. 2010. Computer simulation of dinosaur tracks. Unpublished PhD thesis Thesis. University of Manchester.

Falkingham PL. 2012. Acquisition of high resolution three-dimensional models using free, opensource, photogrammetric software. Palaeontologia Electronica 15:1-15.

Falkingham PL. 2016. Applying Objective Methods to Subjective Track Outlines. In: Falkingham PL, Marty D, Richter A eds. Dinosaur Tracks: The Next Steps. Bloomington: Indiana University Press, 72-81.

Falkingham PL, Bates KT, Avanzini M, Bennett M, Bordy EM, Breithaupt BH, Castanera D, Citton P, Díaz Martínez I, Farlow JO, Fiorillo AR, Gatesy SM, Getty P, Hatala KG, Hornung JJ, Hyatt JA, Klein H, Lallensack JN, Martin AJ, Marty D, Matthews NA, Meyer CA, Milàn J, Minter NJ, Razzolini NL, Romilio A, Salisbury SW, Sciscio L, Tanaka I, Wiseman ALA, Xing LD, Belvedere M. 2018. A standard protocol for documenting modern and fossil ichnological data. Palaeontology 61:469-480. DOI: 10.1111/pala.12373.

Hamilton N. 2015.contoureR: Contouring of non-regular three-dimensional data. Available at https://CRAN.R-project.org/package=contoureR (accessed December 29, 2018). 
Ishigaki S, Fujisaki T. 1989. Three dimensional representation of Eubrontes by the method of moiré topography. In: Gillette DD, Lockley MG eds. Dinosaur tracks and traces. 421425.

Lallensack JN, van Heteren AH, Wings O. 2016. Geometric morphometric analysis of intratrackway variability: a case study on theropod and ornithopod dinosaur trackways from Münchehagen (Lower Cretaceous, Germany). PeerJ 4:e2059.

Leakey MD, Hay RL. 1979. Pliocene footprints in the Laetolil Beds at Laetoli, northern Tanzania. Nature 278:317-323. DOI: 10.1038/278317a0.

Leonardi G, Casamiquela RM, Demathieu GR, Haubold H, Sarjeant WAS. 1987. Glossary and manual of tetrapod footprint palaeoichnology. Brasília: Publicação do Departemento Nacional da Produção Mineral Brasil.

Mallison H, Wings O. 2014. Photogrammetry in paleontology - A practical guide. Journal of Paleontological Techniques 12:1-31.

Matthews NA, Noble T, Breithaupt BH. 2016. Close-Range Photogrammetry for 3-D Ichnology: The basics of photogrammetric ichnology. In: Falkingham PL, Marty D, Richter A eds. Dinosaur Tracks: The Next Steps. Indiana University Press, 28-55.

Romilio A, Salisbury SW. 2011. A reassessment of large theropod dinosaur tracks from the midCretaceous (late Albian-Cenomanian) Winton Formation of Lark Quarry, central-western Queensland, Australia: A case for mistaken identity. Cretaceous Research 32:135-142. DOI: 10.1016/j.cretres.2010.11.003.

Romilio A, Salisbury SW. 2014. Large dinosaurian tracks from the Upper Cretaceous (Cenomanian-Turonian) portion of the Winton Formation, Lark Quarry, central-western Queensland, Australia: 3D photogrammetric analysis renders the 'stampede trigger' scenario unlikely. Cretaceous Research 51:186-207. DOI: 10.1016/j.cretres.2014.06.003. Romilio A, Tucker RT, Salisbury SW. 2013. Reevaluation of the Lark Quarry dinosaur Tracksite (late Albian-Cenomanian Winton Formation, central-western Queensland, Australia): no longer a stampede? Journal of Vertebrate Paleontology 33:102-120. DOI: 10.1080/02724634.2012.694591. 
Sarjeant WAS. 1975. Fossil Tracks and Impressions of Vertebrates. In: Frey RW ed. The Study of Trace Fossils. Springer Berlin Heidelberg, 283-324. DOI: 10.1007/978-3-642-659232_14.

Schlager S. 2017. Morpho and Rvcg - shape analysis in R. In: Zheng G, Li S, Szekely G eds. Statistical Shape and Deformation Analysis. Academic Press, 217-256.

Thulborn RA. 1990. Dinosaur tracks. London, New York: Chapman and Hall.

Thulborn RA. 2013. Lark Quarry revisited: a critique of methods used to identify a large dinosaurian track-maker in the Winton Formation (Albian-Cenomanian), western Queensland, Australia. Alcheringa: An Australasian Journal of Palaeontology 37:312330. DOI: $10.1080 / 03115518.2013 .748482$.

Thulborn RA. 2017. Behaviour of dinosaurian track-makers in the Winton Formation (Cretaceous, Albian-Cenomanian) at Lark Quarry, Western Queensland, Australia: running or swimming? Ichnos 24:1-18. DOI: 10.1080/10420940.2015.1129326.

Thulborn RA, Wade M. 1984. Dinosaur trackways in the Winton Formation (mid-Cretaceous) of Queensland. Memoirs of the Queensland Museum 21:413-517.

Wings O, Lallensack JN, Mallison H. 2016. The Early Cretaceous Dinosaur Trackways in Münchehagen (Lower Saxony, Germany) - 3D photogrammetry as basis for geometric morphometric analysis of shape variation and evaluation of material loss during excavation. In: Falkingham PL, Marty D, Richter A eds. Dinosaur Tracks: The Next Steps. Bloomington: Indiana University Press, 56-71. 


\section{Figure 1}

Procedure of calculating objective outlines of footprints using ornithopod footprint 11-31 from the Lower Cretaceous of Münchehagen, Germany as example.

All six steps are carried out automatically. Axes scales are in meters, and all plots are in zdirection (top view). Plots can be reproduced using the script and the 3D-model provided in Supp. 1 and 2. A. The xyz point cloud is extracted from the submitted PLY mesh, and subjected to principal component analysis (PCA) for fitting to the horizontal plane. B. Contour lines for 30 height levels are extracted based on the point cloud (xyz-coordinates of contours are shown). C. Incomplete and short contours are removed. D. Further contours are removed based on Procrustes distances (i.e., shape similarities). In order to establish correspondence between points of separate outlines, bottleneck points are determined along the outlines (red points). Sections in-between bottleneck points are resampled to equal numbers of equidistant points for each contour, so that each point of a contour has homologous counterparts on the other contours. E. The location of the steepest slope is computed for each set of homologous points. Multiple slopes are taken into account by taking weighted means of the coordinates. F. Elliptical Fourier transforms are used to fit an approximating curve to the succession of points, providing a smooth, continuous outline. 

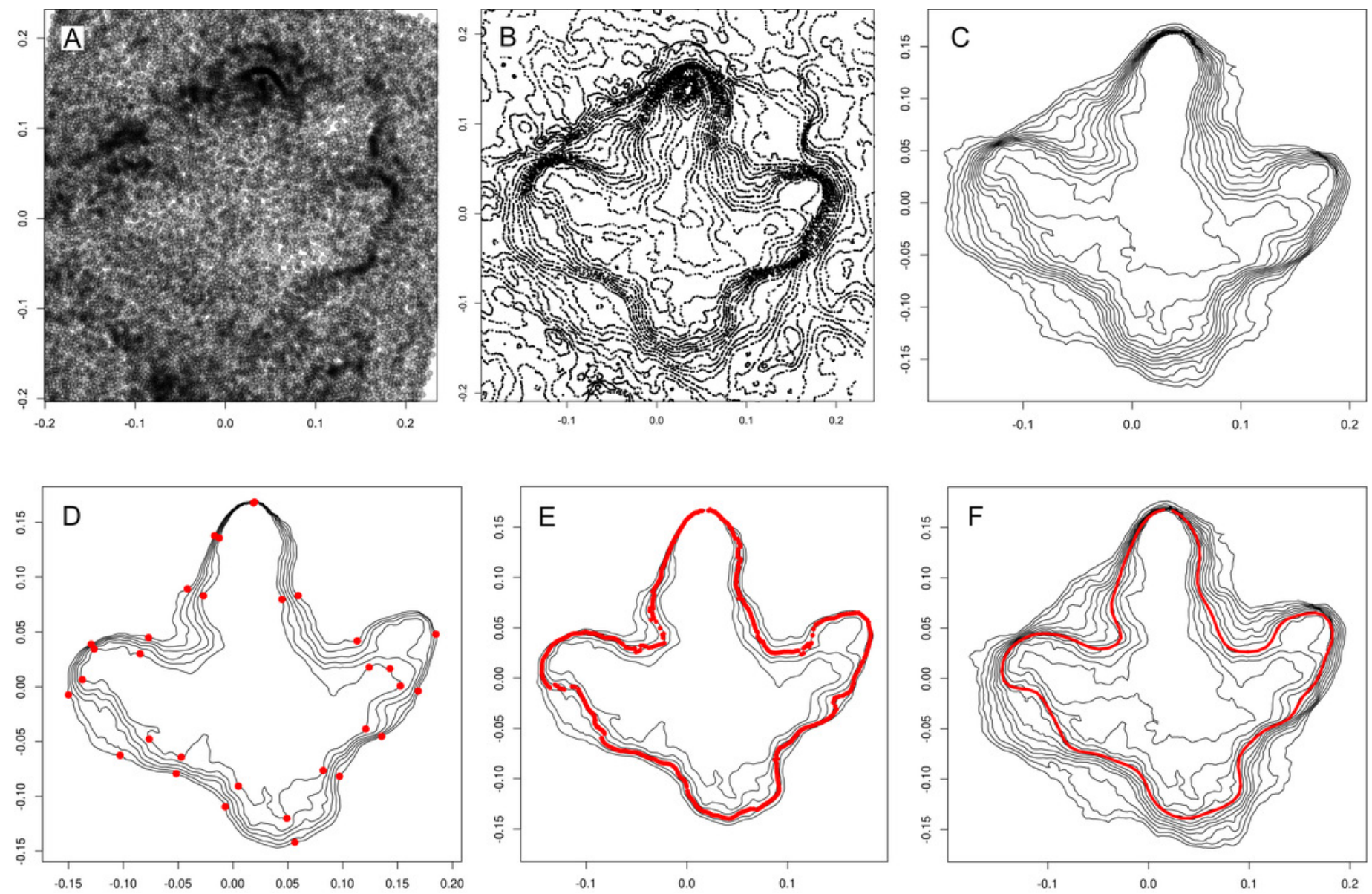


\section{Figure 2}

Objective outlines calculated for various footprints (continuous red lines).

A. Footprint BSY1020-E2 from the Courtedoux-Bois de Sylleux tracksite, Upper Jurassic, Switzerland (Castanera et al., 2018). The generated outline is affected by a crack running transversally through the central digit impression. B. Ornithopod footprint (Caririchnium kyoungsookimi) from the Jindong Formation, South Korea, which is a shallow footprint consisting of three separate impressions. C. Hominin footprint G1-35 from the Pliocene of Laetoli, Tanzania (Bennett 2013). D-F. Footprints of theropod trackway T3 (A: T3/47; B: T3/37) and ornithopod trackway II (I1/35; C) from the Lower Cretaceous Münchehagen locality, Germany (Lallensack, van Heteren \& Wings, 2016). G-H. Tracks 6 (G) and 5 (H) of a large tridactyl trackmaker (specimen QM F10322) from the Upper Cretaceous of Lark Quarry, Australia (Romilio and Salisbury 2014). The interpretive outlines were based on a selected contour line. I. Redrawing of a previous interpretation of track 5 by Thulborn (2017, fig. 5 (3)) $d$, which was drawn based on different assumptions on the trackmaker identity and markedly differs from the generated outline $(\mathrm{H})$. 

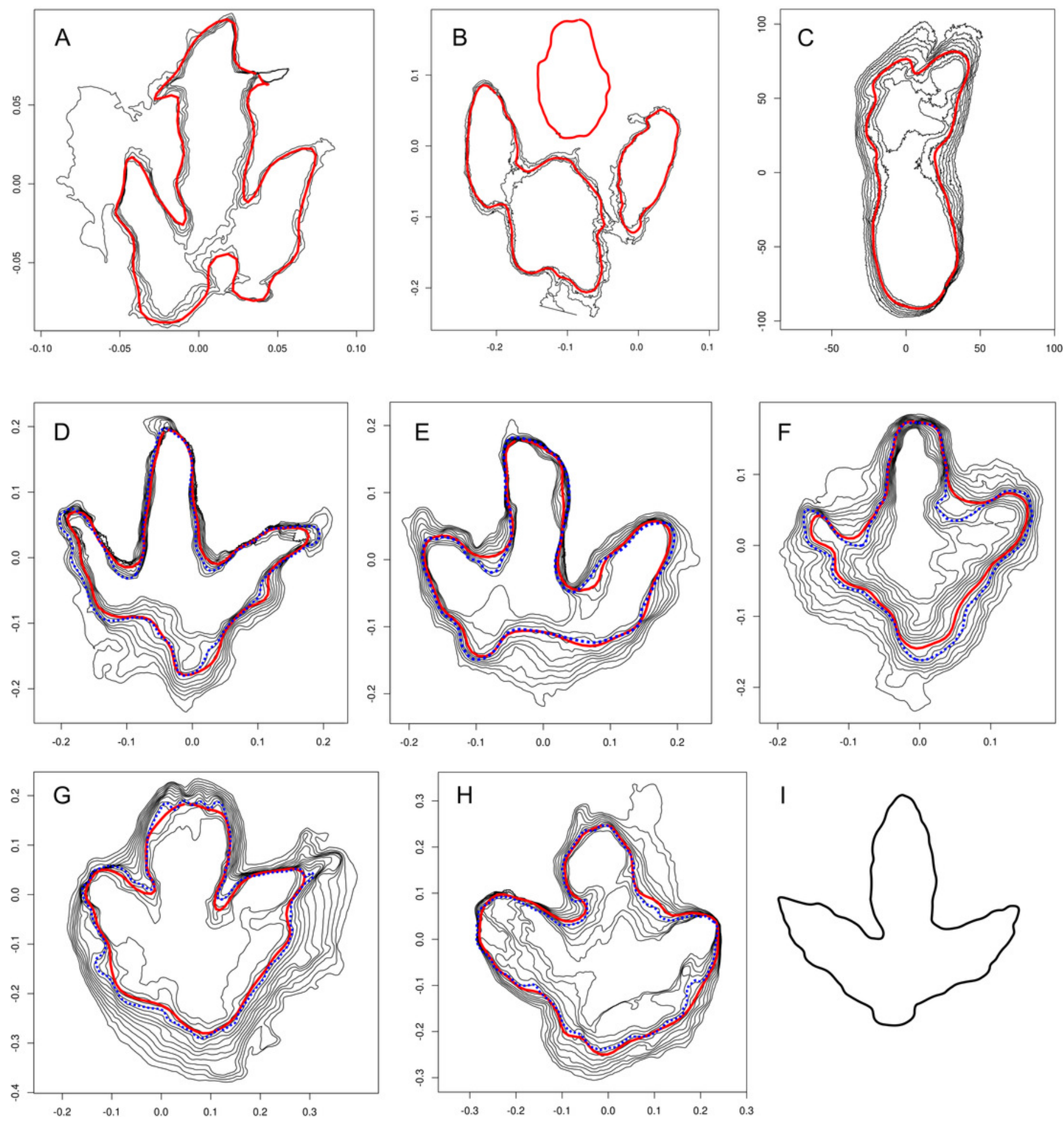


\section{Figure 3}

Quantitative evaluation of the present approach.

A. Footprint T3/23 from Münchehagen, Germany, processed with option to automatically place landmarks enabled. The six generated landmark points are shown as blue crosses; computed digital axes are shown as green lines. B. Procrustes analysis of 13 footprints from trackway T3 of Münchehagen. Black dots represent the mean shape, and grey dots the individual aligned outlines. C. Comparison of the mean shape automatically generated by the script based on the objective outlines (black line, dotted) and that published by Lallensack, van Heteren \& Wings (2016) based on interpretive outlines (red line, solid). D. Comparison of the variability of landmarks, with those derived from the interpretive outlines in blue (left columns) and those produced by the present script in red (right columns). E-F. Sensitivity analysis, comparing the script output of the full-resolution model (E; 196,236 faces, 9.4 MiB) with that of a model of reduced size ( $F ; 5000$ faces, $246.2 \mathrm{KiB})$. 

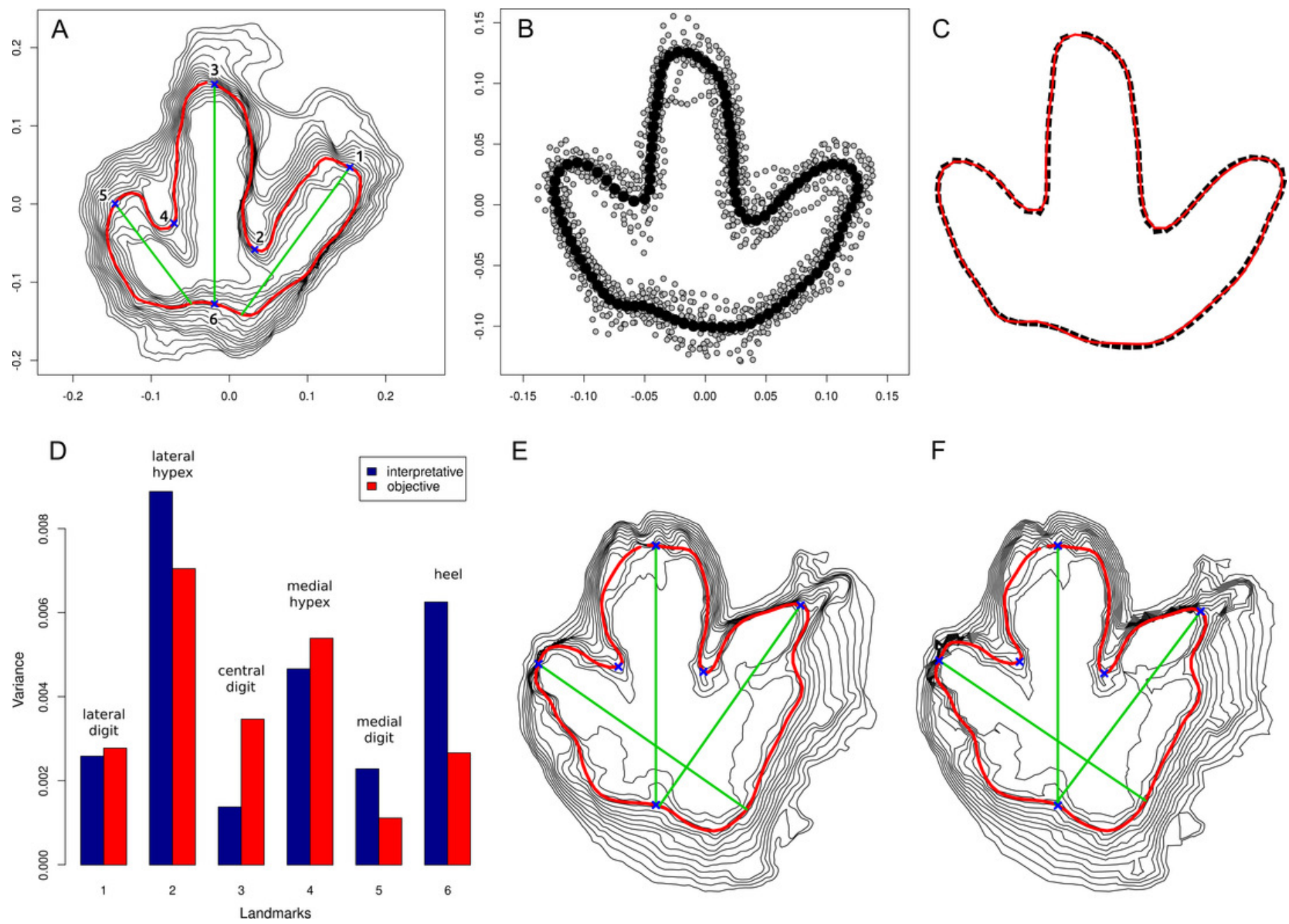

E

F
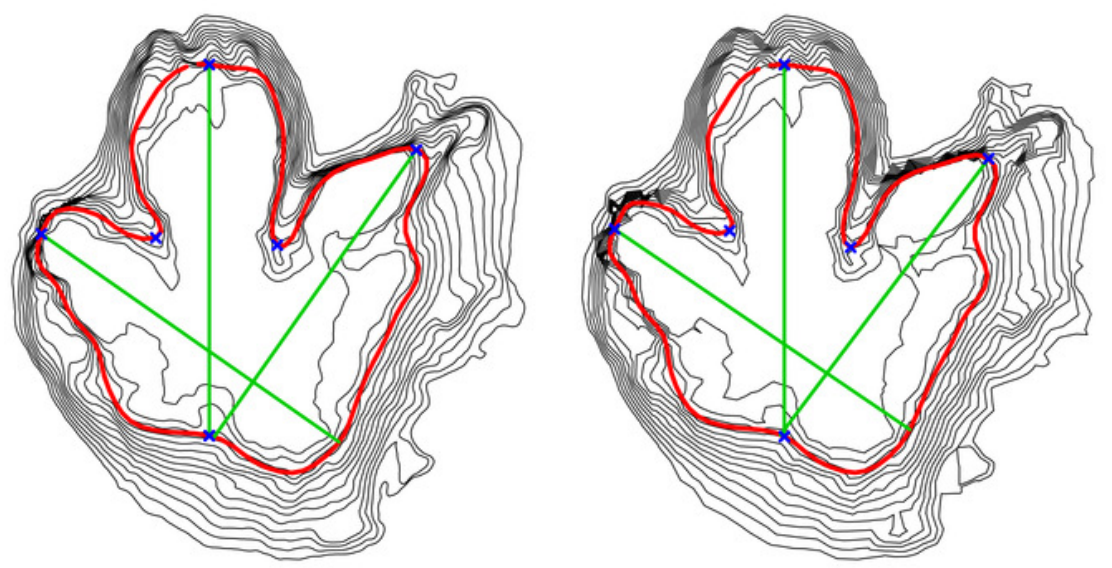